\title{
Benefits of the Therapy With Abexol in Patients With Non- Alcoholic Fatty Liver Disease
}

\author{
Julio Cesar Fernandez-Travieso ${ }^{\mathrm{a}, \mathrm{d}}$, Ivan Rodriguez-Perez ${ }^{\mathrm{b}}$, Caridad Ruenes-Domech ${ }^{\mathrm{c}}$, \\ Jose IIlnait-Ferrer ${ }^{\text {a }}$, Lilia Fernandez-Dorta ${ }^{\text {a }}$, Sarahi Mendoza-Castano ${ }^{\text {a }}$
}

\begin{abstract}
Background: Non-alcoholic fatty liver disease (NAFLD) encompasses a spectrum of diseases ranging from steatosis to steatohepatitis and cirrhosis. Given the increasing incidence of NAFLD and the long-term consequences of this disease, it is important to identify the risk factors and therapeutic measures. Abexol is a mixture of beeswax alcohols with antioxidant, gastro-protective and antiinflammatory effects. The aim was to conduct a pooled analysis of clinical trials data of the effects of Abexol treatment in patients with NAFLD.

Methods: The present analysis includes the data of all patients with NAFLD obtained from medium-term randomized, double-blinded, placebo controlled clinical studies with Abexol. One hundred patients with NAFLD received Abexol (100 mg/day) or placebo for 6 months. Significant changes in the ultrasound analysis of the liver were considered a primary efficacy variable. Secondary endpoints were decreased homeostasis model assessment (HOMA) index and insulin levels, and improved clinical symptoms. Statistical analysis of all data was according to the intention-to-treat method.
\end{abstract}

Results: Both groups were statistically homogeneous at baseline conditions. At 6 months of treatment, the number of Abexol-treated patients exhibiting a normal liver echo pattern on ultrasonography was greater than that of the placebo patients $(\mathrm{P}<0.05)$. Abexol significantly reduced $(\mathrm{P}<0.05)$ insulin levels and HOMA index. The proportion of Abexol patients showing symptom improvement was higher $(\mathrm{P}<0.01)$ than that of the placebo group. Treatments were safe and well tolerated.

Conclusions: Treatment of Abexol during 6 months significantly ameliorates liver fat accumulation and insulin resistances, meanwhile improving clinical evolution in patients with NAFLD. The treatment was safe and well tolerated in these patients.

Manuscript submitted February 21, 2020, accepted March 5, 2020

a National Centre for Scientific Research, Havana, Cuba

${ }^{b}$ Surgical Medical Research Centre, Havana, Cuba

'National Institute of Gastroenterology, Havana, Cuba

${ }^{\mathrm{d} C o r r e s p o n d i n g ~ A u t h o r: ~ J u l i o ~ C e s a r ~ F e r n a n d e z-T r a v i e s o, ~ N a t i o n a l ~ C e n t r e ~ f o r ~}$ Scientific Research, 25 Avenue and 158 st, Cubanacan, Playa, Havana 113000, Cuba.Email: julio.fernandez@cnic.cu

doi: https://doi.org/10.14740/gr1273
Keywords: Abexol; Beeswax; Non-alcoholic fatty deposition liver disease; HOMA index; Insulin levels

\section{Introduction}

Non-alcoholic fatty liver disease (NAFLD) encompasses a spectrum of diseases ranging from steatosis to steatohepatitis and cirrhosis [1-3].

Given the increasing incidence of NAFLD and the longterm consequences of this disease, it is important to identify the risk factors and therapeutic measures, which can help to curtail the progression of this aggressive illness $[4,5]$.

NAFLD results from the presence of fat in the liver parenchyma not accompanied by inflammation, in absence of excessive alcohol consumption and is considered as one of the components of the metabolic syndrome. NAFLD may be influenced, according to more recent data, also by the intestinal microbiome, among other factors like age, gender, the presence of diabetes, and genetic polymorphism [6,7].

NAFLD is usually asymptomatic, being detected incidentally during abdominal ultrasound examinations, liver function tests or by hepatomegaly in the routine clinical examination, although in some cases it is accompanied by diffuse symptoms, such as discomfort in the upper right quadrant of the abdomen or diffuse abdominal discomfort [8].

Abdominal ultrasonography is the most widely used diagnostic method in this disease because it is more economical and standardized, although other imaging modalities have also been used, such as computed tomography and magnetic resonance imaging $[9,10]$.

There is no proven effective therapy for NAFLD. Doubleblinded, randomized, controlled with diet and exercise with a greater number of patients and longer time of treatments including checking the degree of injury are recommended [11].

However, there are limited evidences in relation to some alternative therapies such as decreased body weight, the use of lipid-lowering agents, of sensitizing drugs to insulin, angiotensin converting enzyme inhibitors, nutritional supplements and antioxidants [12-15].

Abexol (D-002), a mixture of primary aliphatic alcohols purified from beeswax (Apis mellifera) [16], is a nutritional supplement that produces moderate anti-inflammatory effects [17] in addition to marked antioxidant $[18,19]$ and gastropro- 
tector effects [20,21].

Randomized, double-blinded, placebo-controlled clinical studies have demonstrated that Abexol improves the ulcer healing process and reduces the ulcer associated symptoms, reduces the frequency of gastrointestinal symptoms in patients under therapy with non-steroidal anti-inflammatory drugs [22, 23], and exerts antioxidant effects in healthy volunteers and in the middle and elderly, reducing lipid peroxidation, markers of protein oxidation and increasing the total antioxidant state of the plasma, as well as being safe and very well tolerated [22-27].

\section{Materials and Methods}

The present analysis shows the data of all patients with NAFLD treated with Abexol during 6 months included in medium-term clinical studies.

The studies were prospective, randomized, double-blinded, placebo controlled and conducted in accordance with the Declaration of Helsinki (as revised in Brazil in 2013) [28], as well as the recommendations of the World Health Organization and the Cuban regulations on Good Clinical Practices. The study protocols were approved by the Ministry of Public Health and by the Ethics Committee in Clinical Research of the Surgical Medical Research Centre and National Institute of Gastroenterology (IRB No. 2018-09-001), as well as registered in the Cuban Public Registry of Clinical Trials.

\section{Patients}

Patients enrolled were of both sexes, aged between 25 and 70 years who attended an outpatient consultation, and were recruited after signing the informed consent, with a history of liver enzyme elevation in routine examinations, obesity or overweight, diabetes, dyslipidemias or with an ultrasound history of liver disease due to non-alcoholic fat deposition.

Patients with ultrasound diagnosis of NAFDL according to standard criteria accepted by the American Gastroenterology Association [29] were included.

Patients with current alcohol consumption, $\mathrm{C}$ and $\mathrm{B}$ virus infection, autoimmune liver disease, hemochromatosis, hepatotoxicity, human immunodeficiency virus (HIV), secondary causes of NAFLD, cirrhotic patients, pregnant or nursing women, and diabetic patients non-compensate were excluded from the study.

Patients receiving treatment that could influence liver function were also excluded.

\section{Treatment}

The studied drugs (Abexol or identical placebo tablets) were taken twice per day (at lunch and dinner) for 6 months, so that treated patients received a dose of Abexol within the range approved in humans $(100 \mathrm{mg} /$ day $)$.

Randomization was computer generated using blocks and $1 / 1$ randomization and treatments were given in identical cod- ed packages accordingly. Consumption of drugs with recognized or suggested antioxidant, liver protective, or hepatotoxic effect was not allowed.

Treatment compliance was assessed by counting the remaining tablets with respect to those that should be consumed in each period. To be acceptable, $\geq 85 \%$ of the tablets scheduled for a period must have been consumed. Diet adhesion was followed by using special chart records filled by patients and by recording body weight at each visit.

\section{Efficacy variables}

The primary outcome measure was improvement of NAFLD ultrasound according to criteria accepted by the American Gastroenterology Association: decrease in hepatic echogenicity based on renal echogenicity; and/or absence of attenuation; and presence of differentiation of the periportal reinforcement and of the vesicular wall due to the decrease of the parenchymal hyper echogenicity, the degree of involvement being standardized by semi-quantitative scale of the degree of hepatic refringement [30].

All patients underwent ultrasonography for liver steatosis after fasting for 10 to $12 \mathrm{~h}$. Ultrasound scans were performed by a trained operator who was blind to participant's group allocations. Steatosis severity was scored using the following scale: 0 (normal), 1 (mild), 2 (moderate), and 3 (severe) [31].

Significant reduction in the values of the insulin resistance index (homeostasis model assessment (HOMA) index) and insulin levels with respect to the baseline values and the group that receives placebo was considered a secondary efficacy variable.

Clinical evaluation was carried out as follows: patients were inquired about the evolution of their symptoms, like liver discomfort or pain, abdominal bloating, and/or nausea. The changes were classified as follows: improved, worsened or unchanged.

\section{Safety and tolerability}

Data from physical examination (bodyweight, body mass index (BMI), pulse rate and arterial pressure), laboratory tests and requests for adverse events (AEs) were included for safety and tolerability analysis.

All undesirable events that newly appeared to patients during the trials, disregarding the cause, were considered as AEs. In accordance with their intensity, AEs were classified as mild, moderate or serious. Mild AEs should not require suspension of study capsules and/or specific treatment of the AEs, moderate AEs should require stopping therapy and/or specific treatment of the AEs, and serious AEs should lead to hospitalisation and/or deaths [32].

\section{Laboratory analysis}

Blood venous samples were drowned from 8:00 to 8:30 am, 
Table 1. Baseline Characteristics of Study Patients

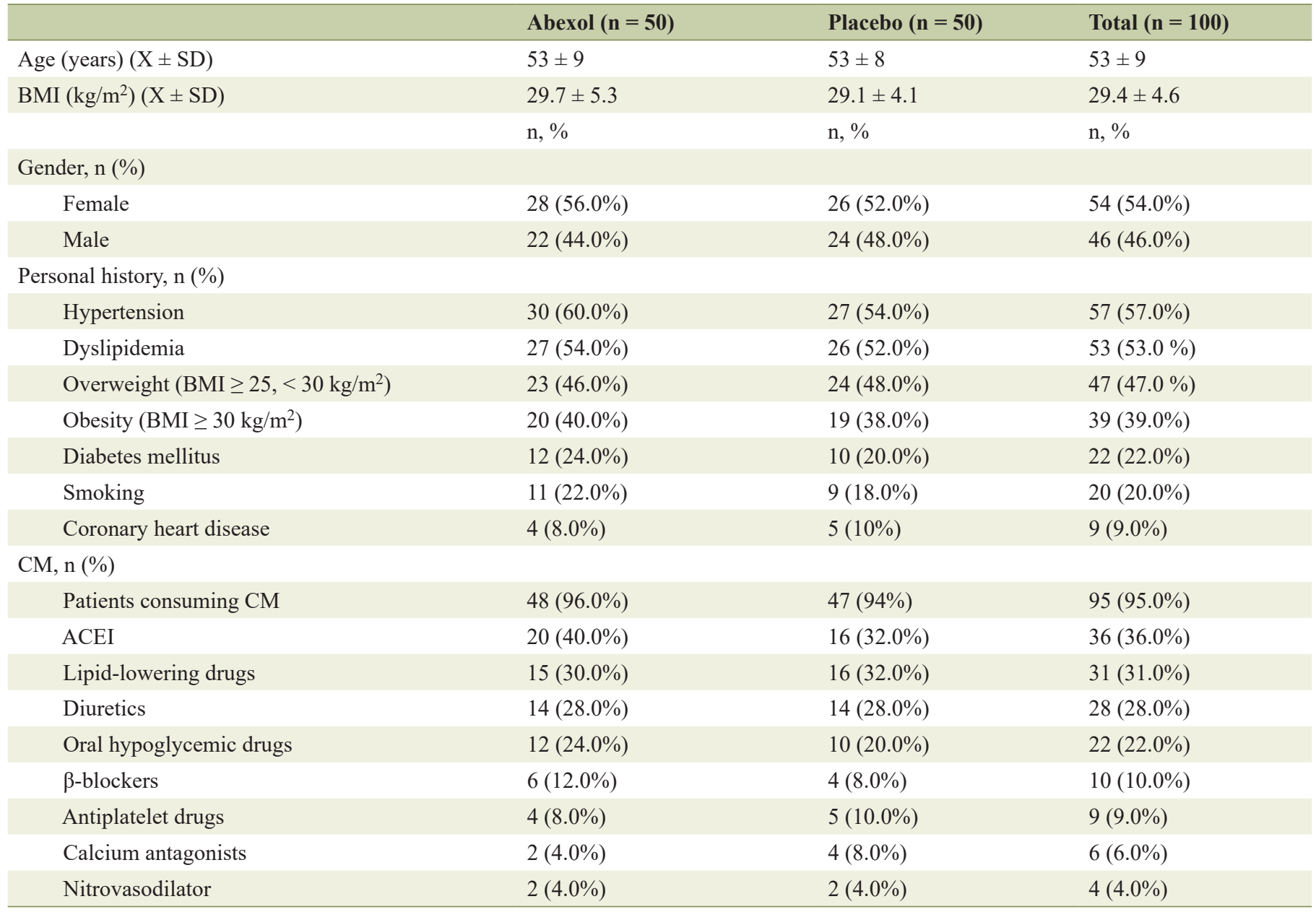

All comparisons were not significant. The table included CM consumed by $\geq$ two patients. BMI: body mass index; CM: concomitant medications; $\mathrm{X}$ : mean; SD: standard deviation; ACEI: angiotensin converting enzyme inhibitor.

after an overnight fasting of $12 \mathrm{~h}$, and aliquots were obtained for laboratory determinations. Liver enzymes (alanine aminotransferase (ALT), aspartate aminotransferase (AST), gamma-glutamyl transferase (GGT)), insulin, serum glucose, and creatinine were determined by enzymatic methods by using reagent kits (Roche, Switzerland) in the Hitachi 912 autoanalyser (Tokyo, Japan).

Insulin levels were determined using radioimmunoassay. The HOMA index was calculated follows:

HOMA index $=$ fasting insulin $(\mu \mathrm{U} / \mathrm{mL}) \times$ fasting blood glucose $(\mathrm{mmol} / \mathrm{L}) / 22.5$

\section{Statistical analyses}

Data analyses were conducted according to intention to treat, including all randomized patients, regardless of studied treatments compliance and the data imputation was performed by the drag method.

The data presented as mean \pm standard deviation. Comparisons of continuous variables were performed using the $t$-test for paired (within group comparisons) and independent samples (between group comparisons). Categorical variables were compares with the Chi-square test. A value of $\alpha=0.05$ was assumed for statistical significance. Comparisons were done with the SPSS 21 system on Windows 10 (SPSS Inc, Chicago, IL, USA).

\section{Results}

\section{Baseline characteristics of the patients studied}

In this study, 106 patients were recruited, with 100 being included in the active treatment phase. Six patients were not included because they had no ultrasonographic evidence of fat infiltration. Table 1 shows the main baseline characteristics of the study population. The groups were statistically homogeneous in all the comparisons made.

The average age of the population studied was 53 years and the most prevalent personal history was arterial hyperten- 
Table 2. Effects on Ultrasonographic Evaluation

\begin{tabular}{|llll|}
\hline Degree of severity & Abexol & Placebo & P value \\
\hline Baseline & & & \\
\hline Normal & 0 & 0 & $\mathrm{~ns}$ \\
\hline Mild & 17 & 18 & $\mathrm{~ns}$ \\
\hline Moderate & 23 & 24 & $\mathrm{~ns}$ \\
\hline Severe & 10 & 8 & $\mathrm{~ns}$ \\
\hline Six months & & & \\
\hline Normal & 14 & 6 & $\mathrm{P}<0.05$ \\
\hline Mild & 16 & 18 & $\mathrm{~ns}$ \\
\hline Moderate & 17 & 21 & $\mathrm{~ns}$ \\
\hline Severe & 3 & 5 & $\mathrm{~ns}$ \\
\hline
\end{tabular}

$\mathrm{P}<0.05$ comparison with placebo $\left(\mathrm{X}^{2}\right.$ test). ns: no significant.

sion (57\%). Other personal antecedents present in the study population $(\geq 20 \%)$ were dyslipidemia $(53 \%)$, overweight (47\%), obesity (39\%), diabetes (22\%) and smoking (20\%).

The $95 \%$ of patients consumed some medication, the most frequent being angiotensin converting enzyme inhibitors (36\%), lipid-lowering drugs (31\%), diuretics (28\%), and oral hypoglycemic drugs $(22 \%)$ as expected according to the number of hypertensive patients, patients with dyslipidemia and diabetic patients.

Of the 100 patients included, 85 completed the treatment, and 15 patients were discharged: three for AEs (placebo group), nine patients for not wishing to continue and three patients for trips abroad. Comparisons between groups were not significant.

The adherence to treatment was satisfactory and comparable between the groups, since the patients consumed $>85 \%$ of the treatment that corresponded to them.

No significant difference between groups in dietary and treatment compliance was observed.

\section{Effects on primary efficacy outcomes}

The results of the ultrasonographic evaluation of the liver are shown in Table 2. At baseline, the stages of steatosis were comparable in both groups, and no patient was classified as normal
$($ score $=0)$. At the end of treatment 14/50 (28\%), Abexol patients exhibited a normal liver echo pattern on ultrasonography versus $6 / 50(12 \%)$ placebo patients $(\mathrm{P}<0.05)$.

\section{Effects on secondary efficacy outcomes}

Abexol treatment significantly reduced insulin levels and the HOMA index by $29.1 \%$ and $26.3 \%$, respectively $(\mathrm{P}<0.05$ as compared to baseline and placebo) (Table 3 or Fig. 1).

At 6 months of therapy, the proportion of Abexol-treated patients who declared clinical improvement of some symptoms $(30 / 50,60 \%)$ was significantly greater $(\mathrm{P}<0.01)$ than that of the placebo group $(4 / 50,8 \%)$, meanwhile more $(\mathrm{P}<$ $0.05)$ placebo patients $(40 / 50,80 \%)$ than Abexol-treated patients $(10 / 50,20 \%)$ declared no change in their symptoms, and only six patients in the placebo group declared that the symptoms had worsened.

\section{Safety and tolerability analysis}

Abexol given for 6 months was safe and well tolerated by the patients. The treatment did not affect physical safety indicators (values not shown for simplicity), nor significantly modified the remaining blood indicators laboratory indicators investigated during the study (Table 4 or Fig. 2) in any of the comparisons made. In addition, individual values remained within normal range.

Nine patients reported mild AEs during the studies, four (8\%) from the Abexol group and five (10\%) from the placebo group (values not shown for simplicity).

\section{Discussion}

The adequate treatment of patients with liver disease due to non-alcoholic fatty deposition should be carried out on the basis of comprehensive and multidisciplinary care; therefore, the changes in lifestyle along with diet, exercise and medications constitute the basic pillars for its management $[4,8,12]$.

In this study, the distribution by sex was homogeneous in both groups, with a greater percentage of women (54\%) than men $(46 \%)$. Although this difference was not significant, it is

Table 3. Effects on Secondary Efficacy Outcomes $(X \pm S D)$

\begin{tabular}{llll}
\hline Treatment & Baseline & Six months & Changes (\%) \\
\hline $\begin{array}{l}\text { Insulin levels }(\mu \mathrm{UI} / \mathrm{mL}) \\
\quad \text { Placebo }\end{array}$ & $70.6 \pm 29.8$ & $73.2 \pm 30.1$ & +3.7 \\
$\quad \begin{array}{l}\text { Abexol } \\
\text { HOMA index }\end{array}$ & $71.4 \pm 28.6$ & $50.6 \pm 31.2^{\mathrm{a}, \mathrm{b}}$ & -29.1 \\
$\quad$ Placebo & $11.5 \pm 5.5$ & $13.3 \pm 8.6$ & +15.7 \\
Abexol & $12.2 \pm 8.2$ & $9.0 \pm 5.1^{\mathrm{a}, \mathrm{b}}$ & -26.3 \\
\hline
\end{tabular}

aP $<0.05$ comparison with baseline ( $t$-test for paired samples); ${ }^{\text {pP }}<0.05$ comparison with placebo ( $t$-test for independent samples). $X$ : mean; SD: standard deviation. 


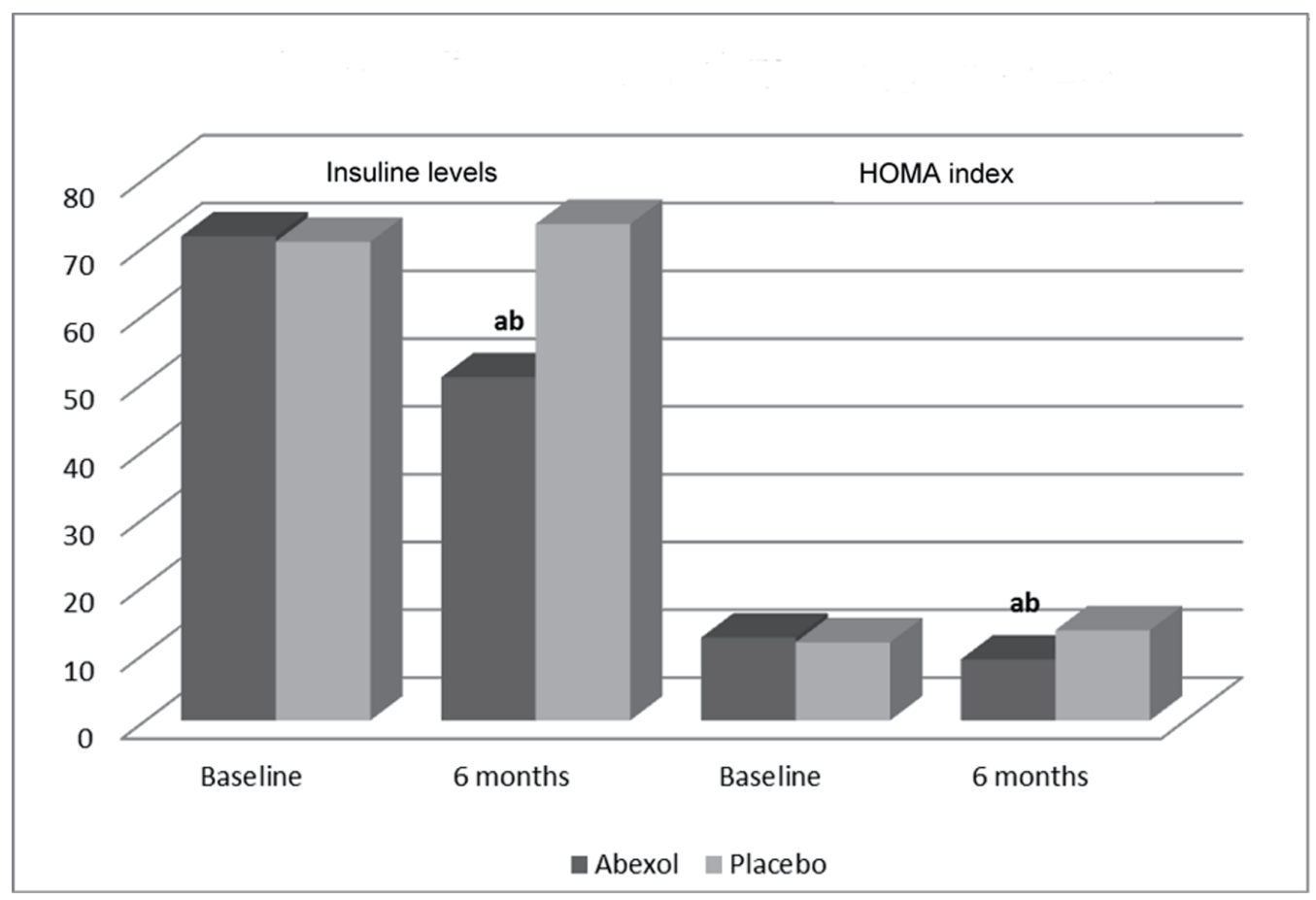

Figure 1. Effects on secondary efficacy outcomes. ${ }^{\text {aP }}<0.05$ comparison with baseline ( $t$-test for paired samples); ${ }^{b} \mathrm{P}<0.05$ comparison with placebo ( $t$-test for independent samples).

partly a reflection of what happens in routine clinical practice, in which women are more motivated to participate in clinical trials and are more disciplined.

The most prevalent personal history among the study patients was the prevalence of obesity + overweight $(86.0 \%)$,

Table 4. Effects on Laboratory Variables $(X \pm S D)$

\begin{tabular}{|clll}
\hline Treatment & Baseline & Three months & Six months \\
\hline AST (U/L) & & & \\
Placebo & $28.1 \pm 10.2$ & $28.9 \pm 10.5$ & $27.3 \pm 11.1$ \\
Abexol & $28.4 \pm 11.3$ & $27.3 \pm 10.8$ & $26.2 \pm 10.7$ \\
ALT (U/L) & & & \\
Placebo & $32.6 \pm 13.4$ & $30.9 \pm 12.5$ & $31.4 \pm 14.7$ \\
Abexol & $31.9 \pm 14.3$ & $30.1 \pm 11.9$ & $29.6 \pm 13.5$ \\
GGT (U/L) & & & \\
Placebo & $41.2 \pm 28.6$ & $39.9 \pm 25.8$ & $40.1 \pm 26.7$ \\
Abexol & $42.1 \pm 26.5$ & $40.3 \pm 23.7$ & $38.9 \pm 24.5$ \\
Glucose (mmol/L) & & & \\
\hline Placebo & $5.4 \pm 1.6$ & $5.5 \pm 1.0$ & $5.0 \pm 1.7$ \\
Abexol & $5.7 \pm 1.4$ & $5.5 \pm 1.2$ & $5.2 \pm 1.1$ \\
\hline Creatinine $(\mu \mathrm{mol} / \mathrm{L})$ & & & \\
\hline Placebo & $75.9 \pm 13.4$ & $76.8 \pm 14.2$ & $75.3 \pm 15.1$ \\
\hline Abexol & $80.6 \pm 18.6$ & $79.1 \pm 14.4$ & $78.6 \pm 14.7$ \\
\hline
\end{tabular}

All comparisons were not significant. X: mean: SD standard deviation; ALT alanine aminotransferase; AST: aspartate aminotransferase; GGT: gamma glutamyl transferase. which coincides with the fact that obesity is the greatest risk factor described for NAFLD. The number of patients with hypertension was also high (57\%) and is consistent with the concomitant consumption of antihypertensive (angiotensin converting enzyme inhibitor (ACEI): 36\%, diuretics: 28\%).

Dyslipidemia was present in 53\% of the patients, of whom 31 patients received lipid-lowering therapy. Type 2 diabetes mellitus frequently coexists in this population, as they share risk factors such as adiposity and insulin resistance. However, the prevalence of type 2 diabetes mellitus is $20-35 \%$ in patients with NALFD $[10,33]$, so that the existence of $22 \%$ in the population studied is within the estimated range.

The efficacy analysis showed satisfactory results for the Abexol treatment evaluated in relation to the primary efficacy variable. At the beginning of the study, all patients presented some degree of steatosis, confirming that they were well included according to the ultrasound performed. At the end of treatment the number of patients $(28 \%)$ that exhibited a normal liver echo pattern on ultrasonography was significantly higher $(\mathrm{P}<0.05)$ than that in the placebo group $(12 \%)$. The results of the ultrasonographic evaluation of the liver showed that the number of patients who managed to reduce echogenicity by at least one degree or remained the same at the end of the study (6 months) too was higher in the Abexol group versus placebo group.

On the other hand, Abexol was effective for lowering insulin resistance as we found a significant decrease in the HOMA index $(26.3 \%)$, an evidence-based surrogate measure of insulin resistance, and insulin (29.1\%) levels after 6 months of treatment. In light of this fact, our results suggest the clinical relevance of the potential use of Abexol for improving insulin 


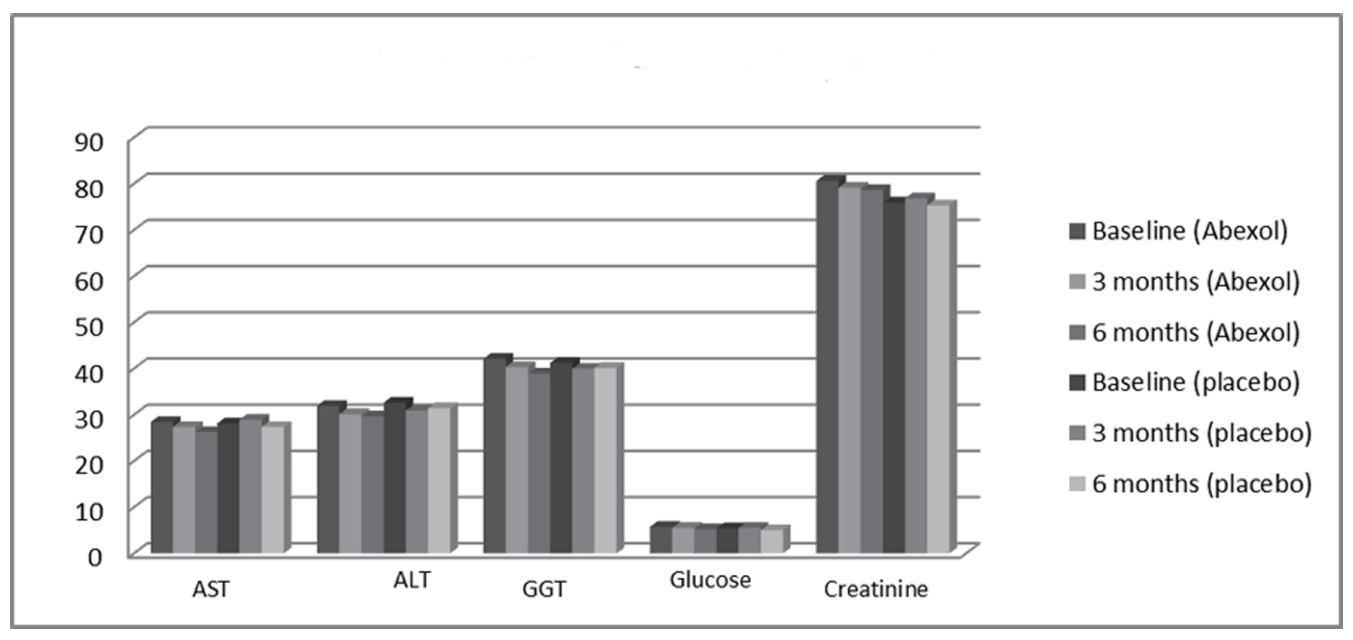

Figure 2. Effects on laboratory variables. All comparisons were not significant. ALT: alanine aminotransferase; AST: aspartate aminotransferase; GGT: gamma glutamyl transferase.

resistance in patients with NAFLD. This effect of Abexol may be explained by the action of triacontanol, its main component, for activating adenosine monophosphate (AMP) kinase, which may lead to metabolic gene regulation and to the AMPmediated activation reducing insulin secretion by pancreatic $\beta$-cells, effect similar to that of metformin, a well-known insulin sensitizer [34].

Overall, the antioxidant effects of Abexol and its action on insulin resistance indicators (HOMA index and insulin levels) may support its efficacy on the ultrasonography reversion of liver fat accumulation.

It is known that inflammation is closely related to NAFLD, so that some inflammation-mediating substances have been investigated as potential diagnostic tools $[33,34]$.

There is experimental evidence of the anti-inflammatory effects of Abexol, which, through the inhibition of the activity of the cycle and lipoxygenase enzymes, causes an inhibition of the synthesis of eicosanoids: prostaglandins and thromboxane by the cyclooxygenase route, and leukotrienes and lipoxins by lipoxygenase pathway. Abexol has an effect on cyclooxygenase (COX), specific on COX-2 and on 5-lipoxygenase (5-LOX), with the latter being involved in the production of leukotrienes B4, which constitutes a potent chemotactic factor for neutrophils, promoting the development of acute inflammation. The inhibition of both enzymes, framing it as dual anti-inflammatory, blocks the synthesis of eicosanoids, and prevents inflammation associated with the development of NAFLD [35-37].

The Abexol treatment did not produce significant changes in the serum levels of liver enzymes (ALT, AST, GGT), although there was a tendency to reduce these values, a very positive effect in this type of patients. At a first glance, this fact seems to be against our efficacy hypothesis, but it should be related to the fact that most patients had normal or relatively low baseline transaminase levels.

Abexol treatments showed a satisfactory safety profile, since there were no significant changes in the physical and laboratory indicators investigated during the study, which coincides with previous results of the clinical evaluation of these treatments $[17,18,22-27]$.

\section{Conclusions}

Abexol for 6 months may ameliorate liver fat accumulation and insulin resistances, meanwhile improving clinical evolution in patients with NAFLD. The treatment was safe and well tolerated in these patients.

Future studies with a greater number of patients with NAFLD and longer treatment are needed to confirm these results.

\section{Acknowledgments}

We thank the Dr. Mirtha Miranda, Dr. Jesus Pinera, BsC. Meilis Mesa (Surgical Medical Research Centre), Dr. Zayli Dorta, Dr. Sacha Lazo, Dr. Marlen Castellanos, BSc. Anaisa Roja, and BSc. Elena Ferrer (National Institute of Gastroenterology, Havana, Cuba), for their contribution in the realization of these studies.

\section{Financial Disclosure}

This study was support by the National Centre for Scientific Research, as part of its research-development projects.

\section{Conflict of Interest}

None to declare.

\section{Informed Consent}

Patients enrolled signed the informed consent. 


\section{Author Contributions}

The authors have been involved in the conception and design of the work, or in the collection of data, or in the analysis and interpretation of the data, as well as in the writing of the article or in its critical review and in the approval of the final version for publication.

\section{Data Availability}

The authors declare that data supporting the findings of this study are available within the article.

\section{References}

1. Sweet PH, Khoo T, Nguyen S. Nonalcoholic fatty liver disease. Prim Care. 2017;44(4):599-607.

2. Ofosu A, Ramai D, Reddy M. Non-alcoholic fatty liver disease: controlling an emerging epidemic, challenges, and future directions. Ann Gastroenterol. 2018;31(3):288295.

3. Lucas C, Lucas G, Lucas N, Krzowska-Firych J, Tomasiewicz K. A systematic review of the present and future of non-alcoholic fatty liver disease. Clin Exp Hepatol. 2018;4(3):165-174.

4. Yoo JJ, Kim W, Kim MY, Jun DW, Kim SG, Yeon JE, Lee JW, et al. Recent research trends and updates on nonalcoholic fatty liver disease. Clin Mol Hepatol. 2019;25(1):111.

5. Leoni S, Tovoli F, Napoli L, Serio I, Ferri S, Bolondi L. Current guidelines for the management of non-alcoholic fatty liver disease: A systematic review with comparative analysis. World J Gastroenterol. 2018;24(30):3361-3373.

6. Benedict M, Zhang X. Non-alcoholic fatty liver disease: An expanded review. World J Hepatol. 2017;9(16):715732.

7. Deeva O, Drapkina V, Ivashkin M, et al. Patients with metabolic syndrome and NAFLD: assessment of obesity and heart fibrosis degree. Journal of Hepatology. 2015;62:S263-S864.

8. Tomic D, Kemp WW, Roberts SK. Nonalcoholic fatty liver disease: current concepts, epidemiology and management strategies. Eur J Gastroenterol Hepatol. 2018;30(10):1103-1115.

9. Li Q, Dhyani M, Grajo JR, Sirlin C, Samir AE. Current status of imaging in nonalcoholic fatty liver disease. World J Hepatol. 2018;10(8):530-542.

10. Obika M, Noguchi H. Diagnosis and evaluation of nonalcoholic fatty liver disease. Exp Diabetes Res. 2012;2012:145754.

11. Cleveland E, Bandy A, VanWagner LB. Diagnostic challenges of nonalcoholic fatty liver disease/nonalcoholic steatohepatitis. Clin Liver Dis (Hoboken). 2018;11(4):98104.

12. Singh S, Osna NA, Kharbanda KK. Treatment options for alcoholic and non-alcoholic fatty liver disease: A review.
World J Gastroenterol. 2017;23(36):6549-6570.

13. Ullah R, Rauf N, Nabi G, Ullah H, Shen Y, Zhou YD, $\mathrm{Fu}$ J. Role of nutrition in the pathogenesis and prevention of non-alcoholic fatty liver disease: recent updates. Int J Biol Sci. 2019;15(2):265-276.

14. Trovato FM, Castrogiovanni P, Malatino L, Musumeci G. Nonalcoholic fatty liver disease (NAFLD) prevention: role of Mediterranean diet and physical activity. Hepatobiliary Surg Nutr. 2019;8(2):167-169.

15. Sigler MA, Congdon L, Edwards KL. An evidencebased review of statin use in patients with nonalcoholic fatty liver disease. Clin Med Insights Gastroenterol. 2018; 11:1179552218787502.

16. Mas R. D-002: Beeswax alcohols. Drugs of the Future. 2001;26:731-744.

17. Carbajal D, Molina V, Valdes S, Arruzazabala ML, Mas R, Magraner J. Anti-inflammatory activity of D-002: an active product isolated from beeswax. Prostaglandins Leukot Essent Fatty Acids. 1998;59(4):235-238.

18. Menendez R, Amor AM, Gonzalez RM, Jimenez S, Mas $\mathrm{R}$. Inhibition of rat microsomal lipid peroxidation by the oral administration of D002. Braz J Med Biol Res. 2000;33(1):85-90.

19. Perez Y, Gonzalez RM, Amor AM, et al. D-002 on antioxidant enzymes in liver and brain of rats. Rev CENIC Cien Biol. 2002;53(1):3-5.

20. Carbajal D, Molina V, Valdes S, Arruzazabala L, Rodeiro I, Mas R, Magraner J. Possible cytoprotective mechanism in rats of D-002, an anti-ulcerogenic product isolated from beeswax. J Pharm Pharmacol. 1996;48(8):858-860.

21. Molina V, Valdes S, Carbajal D, Arruzazabala L, Menendez R, Mas R. Antioxidant effect of D-002 on gastric mucosa of rats with experimentally induced injury. J Med Food. 2001;4(2):79-83.

22. Hano O, Illnait J, Mas R, et al. Effects of D-002, a product isolated from beeswax on duodenal ulcer: a double-blinded placebo-controlled study. Curr Ther Res. 2001;62:394397.

23. Illnait J, Terry H, Mas R, Fernandez L, Carbajal D. Effects of D-002, a product isolated from beeswax, on gastric symptoms of patients with osteoarthritis treated with piroxicam: a pilot study. J Med Food. 2005;8(1):63-68.

24. Menendez R, Mas R, Illnait J, Perez J, Amor AM, Fernandez JC, Gonzalez RM. Effects of D-002 on Lipid Peroxidation in Older Subjects. J Med Food. 2001;4(2):71-77.

25. Menendez R, Mas R, Amor AM, Perez Y, Gonzalez RM, Fernandez J, Molina V, et al. Antioxidant effects of D002 on the in vitro susceptibility of whole plasma in healthy volunteers. Arch Med Res. 2001;32(5):436-441.

26. Illnait J, Rodriguez I, Molina V, et al. Effects of D-002 (beeswax alcohols) on gastrointestinal symptoms and oxidative markers in middle-aged and older subjects. Lat Am J Pharm. 2013;32(2):166-174.

27. Illnait J, Rodriguez I, Mendoza S, Fernandez Y, Mas R, Miranda M, Pinera J, et al. Effects of D-002, a mixture of high molecular weight beeswax alcohols, on patients with nonalcoholic fatty liver disease. Korean J Intern Med. 2013;28(4):439-448.

28. World Medical Association. Declaration of Helsinki- Eth- 
ical Principles for Medical Research Involving Human Subjects (Brazil 2013).

29. Chalasani N, Younossi Z, Lavine JE, Diehl AM, Brunt EM, Cusi K, Charlton M, et al. The diagnosis and management of non-alcoholic fatty liver disease: practice guideline by the American Gastroenterological Association, American Association for the Study of Liver Diseases, and American College of Gastroenterology. Gastroenterology. 2012;142(7):1592-1609.

30. Wilkins T, Tadkod A, Hepburn I, Schade RR. Nonalcoholic fatty liver disease: diagnosis and management. Am Fam Physician. 2013;88(1):35-42.

31. Sanyal AJ, American Gastroenterological A. AGA technical review on nonalcoholic fatty liver disease. Gastroenterology. 2002;123(5):1705-1725.

32. Naranjo CA, Busto U, Seller EM. A method for estimating the probability of adverse drugs reaction. Clin Pharmacol Ther. 1981;30:239-245.
33. Alkhouri N, Poordad F, Lawitz E. Management of nonalcoholic fatty liver disease: Lessons learned from type 2 diabetes. Hepatol Commun. 2018;2(7):778-785.

34. Marchisello S, Di Pino A, Scicali R, Urbano F, Piro S, Purrello F, Rabuazzo AM. Pathophysiological, molecular and therapeutic issues of nonalcoholic fatty liver disease: an overview. Int J Mol Sci. 2019;20(8):1948.

35. Hsieh PS, Jin JS, Chiang CF, Chan PC, Chen CH, Shih $\mathrm{KC}$. COX-2-mediated inflammation in fat is crucial for obesity-linked insulin resistance and fatty liver. Obesity (Silver Spring). 2009;17(6):1150-1157.

36. Perez Y, Oyarzabal A, Ravelo Y, et al. Inhibition of COX and 5-LOX enzymes by D-002 (beeswax alcohols). Current Top Nutraceutical Research. 2014;12(1/2):13-18.

37. Vilar E, Yasells A, Martinez Y, et al. Development and validation of a noninvasive prediction model for non-alcoholic steatohepatitis resolution after lifestyle intervention. Hepatology. 2016;63:1875-1887. 